TITLE:

\title{
The reflexivity of a segre product of projective varieties
}

\author{
$\operatorname{AUTHOR}(\mathrm{S})$ :
}

Fukasawa, Storu

\section{CITATION:}

Fukasawa, Storu. The reflexivity of a segre product of projective varieties. 代数幾何学シン ポジウム記録 2008, 2008: 136-136

ISSUE DATE:

2008

URL:

http://hdl.handle.net/2433/215038

RIGHT: 
THE REFLEXIVITY OF A SEGRE PRODUCT OF PROJECTIVE VARIETIES

(Joint work with Hajime Kaji, [Math. Ann. 342 (2008), 279-289])

Satoru Fukasawa (Waseda University/JSPS)

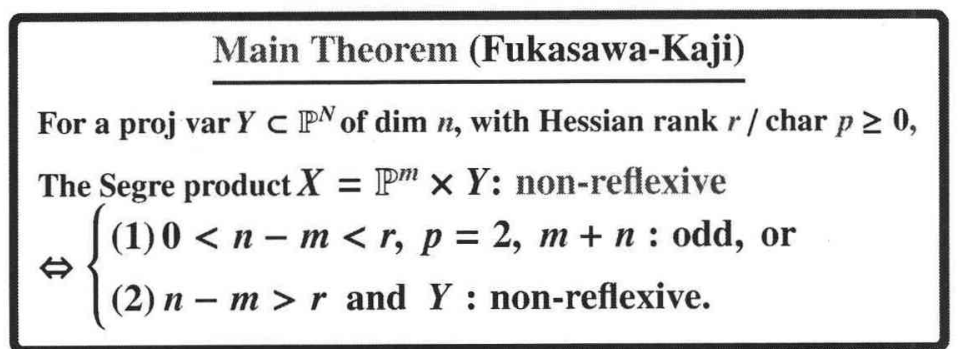

Segre product := Product embedded by Segre embedding

Main Theorem'

$$
\mathbb{P}^{m} \times Y: \text { reflexive } \Leftrightarrow\left\{\begin{array}{l}
\text { (i) } m \geq n, \\
\text { (ii) } 0 \leq n-m<r, p \neq 2, \\
\text { (iii) } 0 \leq n-m<r, p=2, m+n: \text { even } \\
\text { (iv) } n-m=r \text { or } \\
\text { (v) } n-m>r \text { and } Y \text { : reflexive. }
\end{array}\right.
$$

\begin{tabular}{cccc|ccc}
\hline$Y$ & $p$ & $r$ & $\mathbf{R} / \mathbf{N}$ & $\mathbb{P}^{m} \times Y$ & $\mathbf{R} / \mathbf{N}$ & cond. \\
\hline $\mathbb{P}^{n}$ & $\geq 0$ & 0 & $\mathbf{R}$ & $\mathbb{P}^{m} \times \mathbb{P}^{n}(m \geq n)$ & $\mathbf{R}$ & (i) \\
$\mathbb{P}^{1} \times \mathbb{P}^{1}$ & $\neq 2$ & 2 & $\mathbf{R}$ & $\mathbb{P}^{1} \times \mathbb{P}^{1} \times \mathbb{P}^{1}$ & $\mathbf{R}$ & (ii) \\
$\mathbb{P}^{2} \times \mathbb{P}^{2}$ & 2 & 4 & $\mathbf{R}$ & $\mathbb{P}^{2} \times \mathbb{P}^{2} \times \mathbb{P}^{2}$ & $\mathbf{R}$ & (iii) \\
\hline $\mathbb{P}^{1} \times \mathbb{P}^{1} \times \mathbb{P}^{1}$ & 2 & 2 & $\mathbf{N}$ & $\mathbb{P}^{1} \times \mathbb{P}^{1} \times \mathbb{P}^{1} \times \mathbb{P}^{1}$ & $\mathbf{R}$ & (iv) \\
$\mathbb{P}^{1} \times \mathbb{P}^{2}$ & $\geq \mathbf{0}$ & 2 & $\mathbf{R}$ & $\mathbb{P}^{1} \times \mathbb{P}^{1} \times \mathbb{P}^{2}$ & $\mathbf{R}$ & \\
\hline $\mathbb{P}^{1} \times \mathbb{P}^{3}$ & $\geq \mathbf{0}$ & 2 & $\mathbf{R}$ & $\mathbb{P}^{1} \times \mathbb{P}^{1} \times \mathbb{P}^{3}$ & $\mathbf{R}$ & (v) \\
\hline $\mathbb{P}^{1} \times \mathbb{P}^{1}$ & 2 & 2 & $\mathbf{R}$ & $\mathbb{P}^{1} \times \mathbb{P}^{1} \times \mathbb{P}^{1}$ & $\mathbf{N}$ & (1) \\
$\mathbb{P}^{1} \times \mathbb{P}^{2}$ & 2 & 2 & $\mathbf{R}$ & $\mathbb{P}^{2} \times \mathbb{P}^{1} \times \mathbb{P}^{2}$ & $\mathbf{N}$ & \\
\hline$F_{d}(d \equiv 1 \bmod p)$ & $>0$ & 0 & $\mathbf{N}$ & $\mathbb{P}^{m} \times F_{d}(m<n)$ & $\mathbf{N}$ & (2) \\
\hline
\end{tabular}

TABLE: The reflexivity of Segre products of projective varieties R:= Reflexive / N:= Non-Reflexive $F_{d}$ : Fermat hypersurf. of $\operatorname{deg} d$ in $\mathbb{P}^{n+1}$

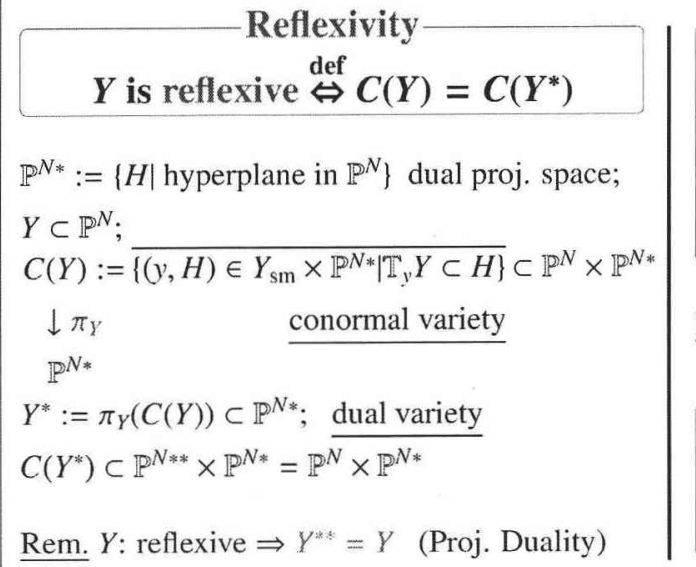

Hessian Rank $r$

$$
r=\operatorname{rank}\left[\frac{\partial^{2}(h \mid Y)}{\partial y_{i} \partial y_{j}}\right]_{i, j}
$$

$(y, H) \in C(Y)$ : general pt,

$h$ : rational function defining $H$,

$\left(y_{i}\right)$ : local coord. of $Y$ at $y$.

$\underline{\mathrm{Rem}} . \operatorname{rank} d \pi_{Y}=N-1-(\operatorname{dim} Y-r) \leq \operatorname{dim} Y^{*}$

Monge-Segre-Wallace Criterion $Y$ is reflexive.

$\Leftrightarrow \pi_{Y}: C(Y) \rightarrow Y^{*}$ is generically smooth.

$\Leftrightarrow \operatorname{rank} d \pi_{Y}=\operatorname{dim} Y^{*}$

\section{$\underline{\text { PROOF OF MAIN THEOREM }}$ \\ - Determine rank $d \pi_{X}$ and $\operatorname{dim} X^{*}$.}

- Use Monge-Segre-Wallace Criterion.

\section{APPLICATION}

Any Non-reflexive example of a Segre product gives a Negative Answer to Kleiman-Piene's question for Gauss maps.

Rem. The Gauss map of any Segre product is gen. smooth onto its image. 